\title{
Nondestructive evaluation of Polymer Coating Structures on Pharmaceutical Pellets using Full Field Optical Coherence Tomography
}

CHEN LI, ${ }^{1}$ J. AXEL ZEITLER, ${ }^{2}$ YUE DONG, ${ }^{1}$ YAO-CHUN SHEN, ${ }^{*}$

${ }^{1}$ Department of Electrical Engineering and Electronics, University of Liverpool, Brownlow Hill, Liverpool L69 3GJ, UK

${ }^{2}$ Department of Chemical Engineering and Biotechnology, University of Cambridge, Pembroke Street, Cambridge, CB2 3RA, UK

* corresponding author: y.c.shen@liverpool.ac.uk

\begin{abstract}
Full field optical coherence tomography (FF-OCT) using a conventional LED light source and a CMOS camera has been developed for characterising coatings on small pellet samples. A set of en-face images covering an area of $700 \mu \mathrm{m} \times 700 \mu \mathrm{m}$ were taken over a depth range of $166 \mu \mathrm{m}$. The three-dimensional structural information, such as the coating thickness and uniformity, was subsequently obtained by analysis of the recorded en-face images. Drug loaded pharmaceutical sustained release pellets with two coating layers and of a sub-millimetre diameter were studied to demonstrate the usefulness of the developed system. We have shown that both coatings can be clearly resolved and the thickness was determined to be $40 \mu \mathrm{m}$ and $50 \mu \mathrm{m}$ for the outer and inner coating layers, respectively. It was also found that the outer coating layer is relatively uniform whilst the inner coating layer has many particle-like features. X-ray Computed Microtomography $(\mathrm{X} \mu \mathrm{CT})$ measurements carried out on the same pellet sample confirmed all these findings. The presented FF-OCT approach is inexpensive and has better spatial resolution compared to other non-destructive analysis techniques such as terahertz pulsed imaging, and is thus considered advantageous for the quantitative analysis of thin coatings on small pellet samples.
\end{abstract}

Keywords: optical coherency tomography (OCT), coating, imaging methods, pellet, excipients

\section{INTRODUCTION}


An ever increasing number of modified release technologies are available to the pharmaceutical industry for the purpose of improving drug therapy via oral administration [1]. Small sized pellets of sub-millimetre diameter have been used to control the release rate of active pharmaceutical ingredients (APIs) in the human body. They are designed to be coated and filled into capsules or compressed into other solid dosage form. The use of pellets allows a more even spread of drug after the dissolution of capsule shell and thus ensures the drug absorption within gastrointestinal tract [2] as well as avoiding dose-dumping problems due to local defects in the coating structure which are much more severe in a monolith as opposed to a multi-particulate release system. Depending on the required dissolution profile, the API can be contained in the pellet core, or directly coated onto a core bead typically made of sugar or polymer excipients. By using sophisticated coatings such as enteric coatings and complex layer structures applied on pellets it is possible to direct and extend the release time to attain full therapeutic efficiency. As the coating quality has a direct impact on the therapeutic efficiency, there is a critical need for analytical techniques to non-destructively assess the coating structures on pharmaceutical pellets.

The current quality control procedures during film coating processing generally evaluate the coating quality of pellets in terms of the average weight gain [3]. The measurement is nonspecific and the weight gain may fail to reflect the coating structure of sample [4]. Investigation into the physical characteristics of pellets is commonly undertaken with microscopy techniques. Images are generally recorded from cross-section cuts of pellets and coating thicknesses are evaluated by measuring the distance between manually marked points on the coating borders [5]. However, this method is very time consuming, it is destructive and can induce artefacts due to plastic deformation during the preparation of cross-sections [6]. Near infrared (NIR) spectroscopy, which is a non-destructive method, has long been used to evaluate pellet coatings in the pharmaceutical industry. However, NIR spectroscopy is inherently an indirect method since it needs additional reference techniques to build a calibration model [7]. Terahertz pulsed imaging (TPI) has also been demonstrated as a powerful method for the non-destructive evaluation of coatings on large pharmaceutical tablet [8-10]. It is an excellent technique to study the coating structure of relatively large pharmaceutical tablets due to its high penetration depth. For small pellets such as the ones studied here which make up the bulk of pharmaceutically used pellets, however, TPI cannot comprehensively resolve the layer structures due to its inherently low lateral resolution, which 
is fundamentally limited by the relatively large wavelength of terahertz radiation (e.g., $300 \mu \mathrm{m}$ at $1 \mathrm{THz}$ ), thus leading to strong scattering from sub-millimetre size pellets.

Recently, we and other groups have demonstrated that optical coherence tomography (OCT) can be used for characterizing the coating thickness of pharmaceutical tablets $[11,12]$ and for imaging pharmaceutical tablets [13]. Tablet coatings with a layer thickness range of $10-60 \mu m$ were measured quantitatively [11]. In all these studies, single point frequency domain OCT systems were used where the depth profile (A-scan) at a given transverse location was obtained using a focused probe beam from a broadband light source. A succession of these A-scans along one dimension of the transverse plane provides a 2D cross section map (B-scan). A full 3D volume is obtained by collecting successive B-scans. Therefore a full OCT measurement requires electromechanically scanning either the sample or the OCT optics in two lateral dimensions. For small pellet samples, however, this approach proves to be difficult and inefficient to perform OCT measurements because of the small size (pellet diameter is less than $1 \mathrm{~mm}$ ).

In this work, we report the development of a full-field OCT (FF-OCT) system for characterising small size pellets. Our FF-OCT system uses an inexpensive conventional infrared LED as the broadband light source and a complementary metal-oxide semiconductor (CMOS) camera as the detector. The measurement is done in parallel. The sample is full-field illuminated and en-face imaged with the CMOS camera, hence eliminating the need for an electromechanical lateral scan. We demonstrate that small sized pharmaceutical pellets can be imaged easily using the presented FF-OCT system, with a high spatial resolution of $3.6 \mu \mathrm{m}$ and $11 \mu \mathrm{m}$ in axial and lateral directions respectively.

\section{EXPERIMENTAL}

\section{FF-OCT Imaging Setup}

Figure 1 shows the schematic diagram of a table-top FF-OCT system which was based on a Michelson interferometer. Broadband light from a conventional infrared LED source ( $\lambda_{0}=850 \mathrm{~nm}, \Delta \lambda=90 \mathrm{~nm}$ ) was first split into reference and sample arms by a non-polarizing 50/50 beam-splitter. Light back scattered by the sample was recombined with the light reflected off the reference mirror at the beam splitter and finally captured with a CMOS camera (Firefly MV, Point Grey). Interference would occur when the optical path length difference between the reference and sample arms is within the coherence length of the light source. For a full OCT measurement, a series of en-face images was acquired at a rate of 
120 frames per second while the reference mirror on a motorised stage was moving at a constant speed of $3 \mu \mathrm{m}$ per second. This resulted in a fixed $25 \mathrm{~nm}$ depth interval between successive en-face images. Each en-face image is composed of $180 \times 180$ pixels (about $4 \mu m \times 4 \mu m$ per pixel) covering a sample area of $700 \mu m \times 700 \mu m$. The stage was scanned over a depth of $166 \mu m$, thus the system eventually acquired a full OCT data cube covering a volume of $700 \mu m \times 700 \mu m \times 166 \mu m$.

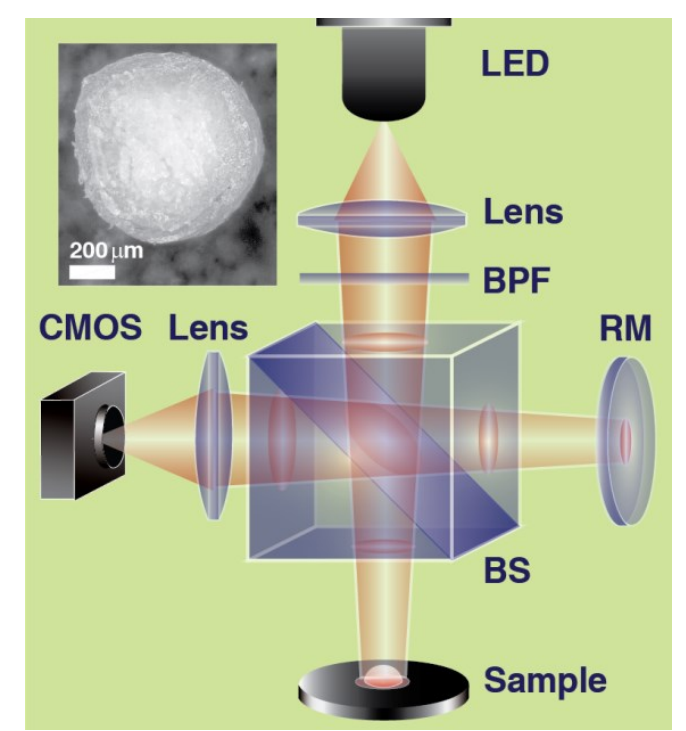

Figure 1 shows the schematic diagram of the FF-OCT system. LED: infrared LED light source; BPF: band-pass filter; BS: beam- splitter; RM: reference mirror. The inset shows the microscopy image of a cross-section through a sample pellet. The inset shows the microscope image of the cross-section through a pellet sample which is spherical with a diameter of about $850 \mu m$.

\section{X-ray Computed Microtomography $(\mathrm{X} \mu \mathrm{CT})$}

The same pellet sample was analysed using a SkyScan1172 high resolution $\mathrm{X} \mu \mathrm{CT}$ scanner (Bruker-microCT, Kontich, Belgium). The sample was imaged at an isotropic voxel resolution of $1.2 \mu \mathrm{m}$ over a total of 5 hour acquisition time and a subsequent image reconstruction time of about 4 hours, using the NRecon program (version 1.6.8.0, Bruker-microCT, Kontich, Belgium).

The sample used in the presented work was a pellet with two coating layers. The core is microcrystalline cellulose (MCC) sphere (CP507). The inner coating is a drug loaded layer containing $10 \%$ active pharmaceutical ingredient (API) and 90\% hydroxypropyl methylcellulose (HPMC). The outer coating is ethyl cellulose and hydroxypropyl cellulose 
combination. As shown in the inset of the Figure 1, the coated pellet is approximately spherical with an outer diameter of about $850 \mu \mathrm{m}$.

\section{RESULTS}

Figure 2 (a) shows a typical raw interferogram signal which is extracted from the volumetric OCT data cube recorded for a coated pellet sample. The tomography signal (Figure 2 (b)), which is a more intuitive and better representation of the sample depth profile, is calculated by demodulating the raw interferogram signal using a Hilbert Transform [14]. A mean refractive index of 1.5 was used in all calculations. Several interference patterns are clearly visible. The first major interference feature at $z=0$ corresponds to the reflection/scattering from the pellet surface. The full width at half maximum (FWHM) of the envelope of this main interferogram feature, which corresponds to the axial resolution achieved here, is determined to be $3.6 \mu \mathrm{m}$, as shown in the inset of Figure 3 (a). Note that the axial resolution of an OCT system is ultimately determined by the coherence length of the light source and can be calculated using the following equation: [15]

$$
l_{c}=0.44 \frac{\lambda_{0}^{2}}{\Delta \lambda}
$$

where $\lambda_{0}=850 \mathrm{~nm}$ is the centre wavelength and $\Delta \lambda=90 \mathrm{~nm}$ is the spectral bandwidth of the LED light source used in our experiment. The axial resolution is thus theoretically calculated to be $3.5 \mu \mathrm{m}$ which agrees very well with the measured resolution of $3.6 \mu \mathrm{m}$. 


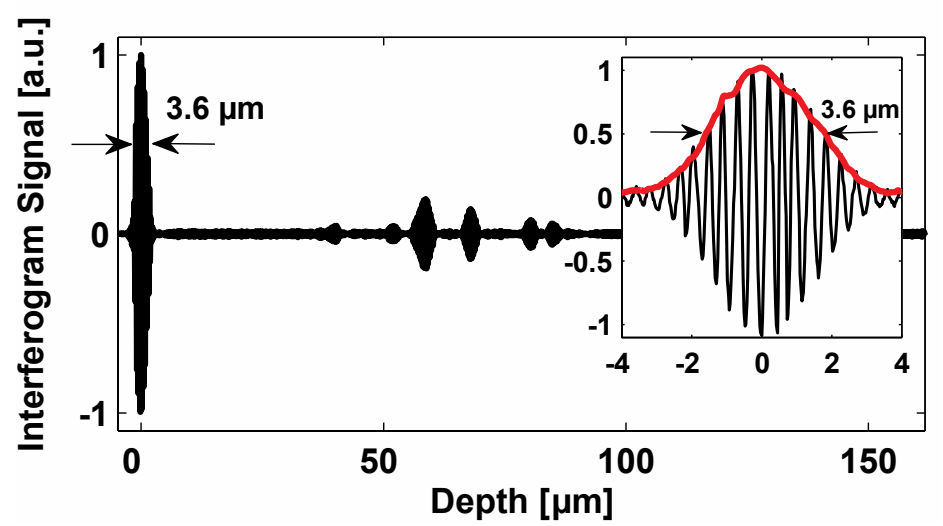

(a)

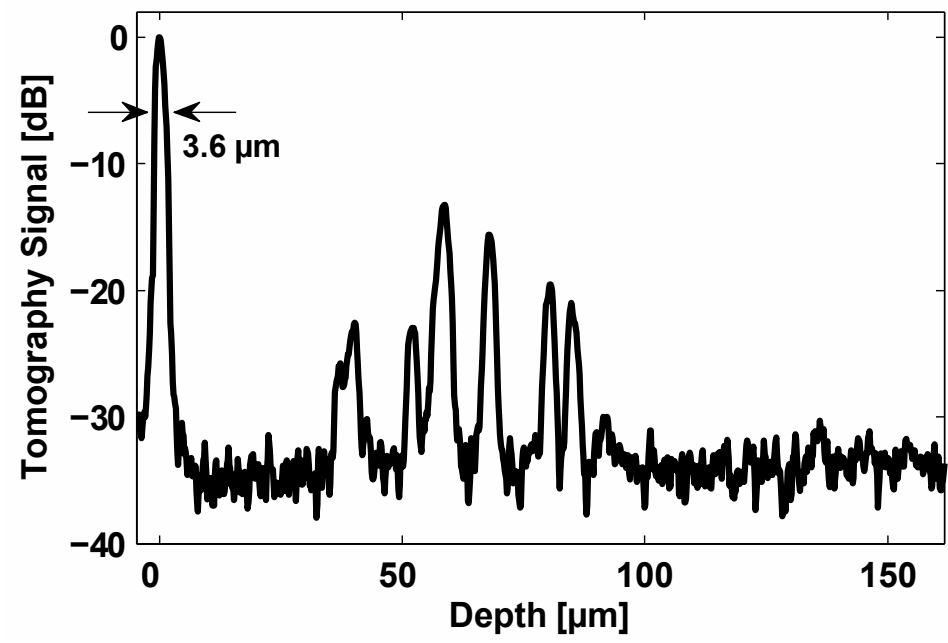

(b)

Figure 2: A typical raw interferogram signal acquired from a two-layer pellet (a), and its corresponding tomography signal in decibel (b). The inset of Figure 1 (a) shows an enlarged view of the first main peak, with the envelope (in red) showing the demodulated tomography signal. The achieved axial resolution is $3.6 \mu \mathrm{m}$.

A set of cross-sectional images (B-scan images) was reconstructed from the recorded volumetric OCT data cube. Figure 3 (a) shows one such B-scan image of the pellet sample, revealing its internal structures. A scale bar calibrated in decibel units is included. Black corresponds to the highest signal and white corresponds to the lowest. As shown in Figure 3 (a), two coating layers were distinguishable (two yellow dashed lines were used to indicate the interface): the outer layer has a mean thickness of about $40 \mu \mathrm{m}$ whilst the inner layer has a mean thickness of $50 \mu \mathrm{m}$. In addition, Figure 2 (b) and Figure 3 (a) also show that the first $40 \mu m$ thick layer is relatively "clean", e.g., no peaks in the tomography signal and no particle like features in the B-scan image in the first $40 \mu \mathrm{m}$ below the surface, indicating that the outer coating layer is fairly uniform. In contrast, the region of $41-90 \mu m$ below the 
pellet surface is dominated by multiple peaks in the tomography signal and particle like features in the B-scan image, suggesting that the inner coating layer is not uniform at all. The particle like features could be API particles that are dispersed in a HPMC matrix. Note that the presence of air bubbles in the coating layer could also lead to the particle-like features observed here. Thus further studies are needed in order to understand this fully.
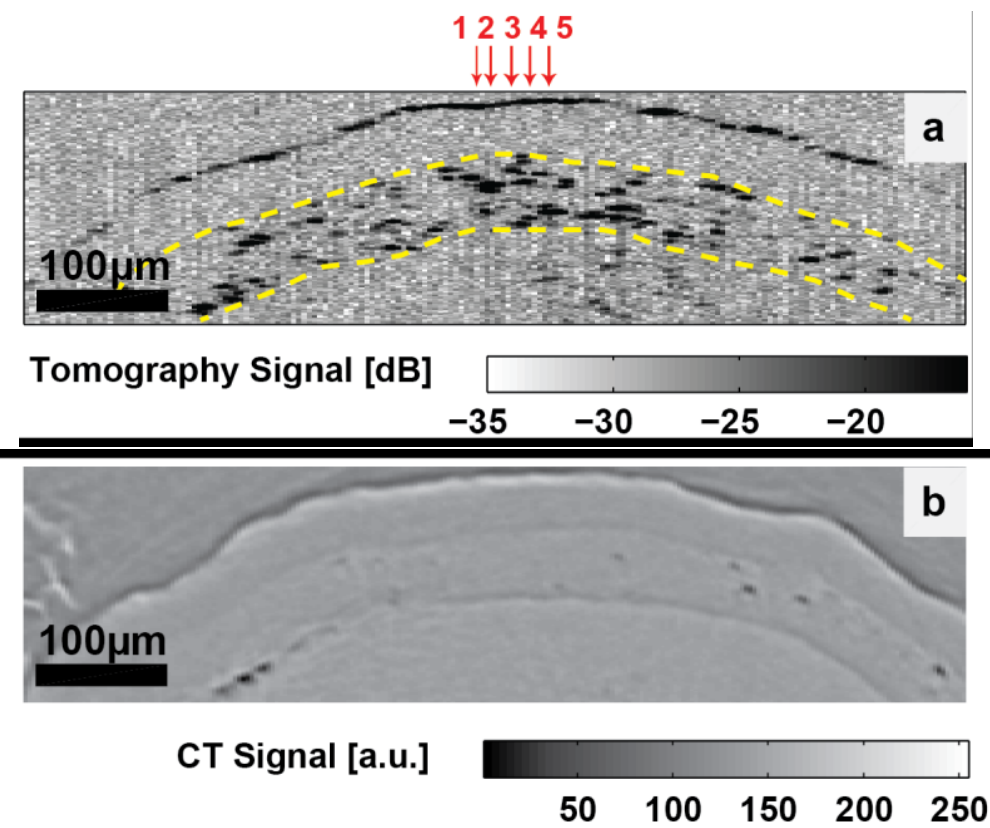

Figure 3: A typical B-scan map (cross-sectional image) of a pellet sample obtained non-destructively using FF-OCT (a) and $\mathrm{X} \mu \mathrm{CT}$ (b). In (a) two dashed yellow lines were used to indicate the interfaces. The red arrows mark the position of the five signal traces plotted in Figure 4 (a). For comparison, both B-scan maps cover approximately an area of $700 \mu m \times 166 \mu m$ (lateral $\times$ axial). Note that the images do not correspond to the exact same location in each sample but are representative of the morphology in each case.

In order to validate the OCT results we performed the $\mathrm{X} \mu \mathrm{CT}$ measurement on the same pellet. Figure 3 (b) shows the resultant $\mathrm{X} \mu \mathrm{CT}$ b-scan image, confirming that the pellet sample indeed has two coating layers and the inner coating layer has many particle-like features with a transversal size of $\sim 9 \mu \mathrm{m}$. The mean thickness of each layer was manually determined as the averaged thickness of 40 selected points from the B-scan map, and the obtained values were $40 \mu \mathrm{m}$ and $50 \mu \mathrm{m}$, for the outer and inner coating layer, respectively. We can conclude that the FF-OCT results are consistent with those of $\mathrm{X} \mu \mathrm{CT}$. 


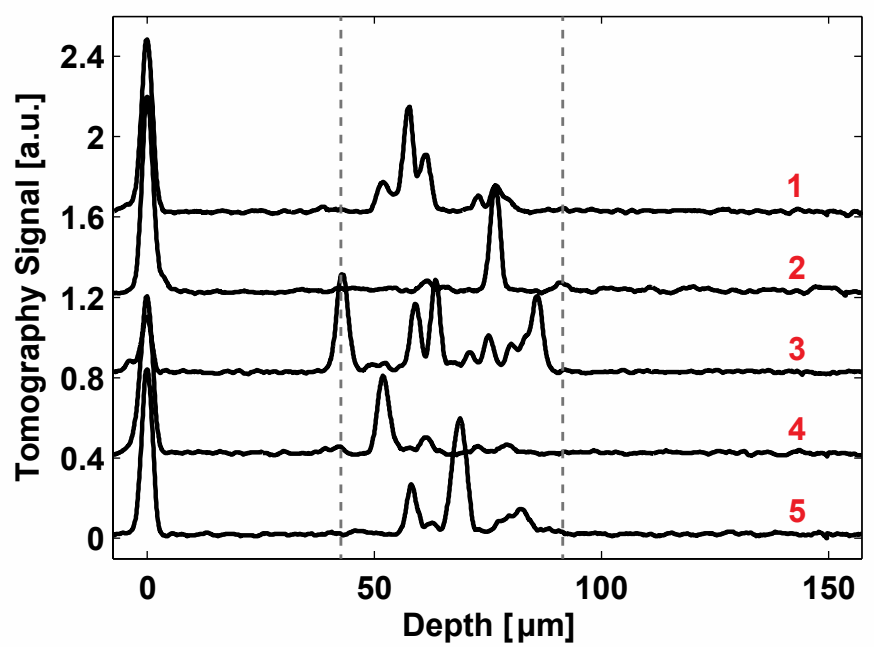

(a)

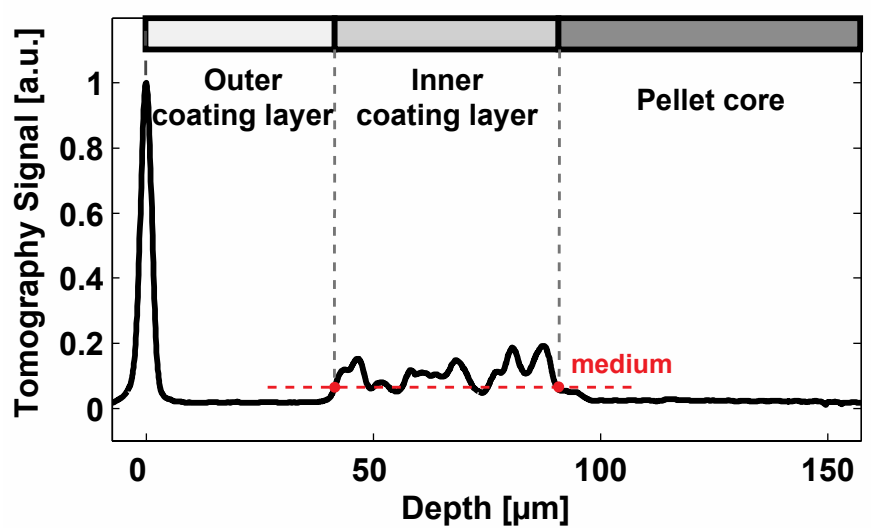

(b)

Figure 4: (a) Five randomly selected signals from the region of interest (ROI), showing the inhomogeneity of the inner coating layer. The position of these pixels is also identified using red arrows in Figure 3 (a). (b) The mean signal obtained by averaging 200 signals within the ROI. The two red-dot indicate the starting and ending position of the inner coating where the tomography signal corresponds the medium value of the OCT signal of the inner coating layer. It quantitatively illustrates that $0-40 \mu \mathrm{m}$ depth region is the outer coating layer, and $41-90 \mu \mathrm{m}$ is the inner coating layer.

One of the key metrics to characterise the coating structure of the pellet is the mean thickness of the respective coating layers. We note that for this specific type of pellet the boundary between neighboring coating layers is not always clearly visible in the b-scan map (Figure 3(a)), or in the optical microscope images (inset of Figure 1). As further illustrated in Figure 4 (a), the coating thickness cannot be precisely determined from a single OCT tomography signal. In order to determine the coating thickness in a robust and automatic way, we propose here to use the mean OCT tomography signal over a wide central volume to calculate the 
coating thickness. The region of interest (ROI) is illustrated in Figure 5 (a). About 200 neighboring OCT tomography signals from this region (here of a diameter of $68 \mu \mathrm{m}$ ) were used to calculate a mean tomography signal. The area is chosen to provides sufficient number of tomography signals whist the measurement error introduced by the surface curvature is still within an acceptable range (e.g., the thickness error is smaller than $0.2 \mu \mathrm{m}$ for a $40 \mu \mathrm{m}$ thick coating layer, referred to Figure 5 (b)). Figure 4 (a) shows five randomly selected tomography signals within this region, confirming that particle like features or air bubbles appear randomly distributed in the range of approximately 41-90 $\mu \mathrm{m}$. Figure 4 (b) shows the mean tomography signal over the ROI by averaging all 200 OCT tomography signals. As shown in this figure, the tomography signal in the range of approximately $41-90 \mu m$ has non-zero values, and its medium value was subsequently used to determine the starting and the end position of the inner coating layer (e.g., at a depth of $41 \mu \mathrm{m}$ and $90 \mu \mathrm{m}$, respectively, as indicated by the two red-dots.). Consequently the mean coating thickness can now be easily determined to be $40 \mu \mathrm{m}$ and $50 \mu \mathrm{m}$ for the outer and inner coating layers, respectively. It should be pointed out that for sample with an uneven structures such as the drug loaded layer in the pellet sample studied in this work, the proposed method can be used for calculating the mean coating thickness although it is not possible to obtain a coating thickness map of the sample. For tablet with uniform coating and core structures, the measured OCT tomography signals show distinct peaks and the resultant B-scan maps show uniform coatings with clear boundaries, as reported in previous studies [11-13]. These distinct peaks correspond to the change in refractive index at the air/surface and coating/core interface of a tablet sample. Consequently the coating thickness at each pixel (rather than the mean coating thickness) could be directly determined as the peak position difference (divided by the refractive index of the coating), in a similar way as in the coating analysis by using terahertz pulsed imaging [8,9]. This allows the coating thickness map to be subsequently constructed as well. We can conclude that FF-OCT can be used to quantify the coating layer thickness and for characterising the uniformity of the coating layer.
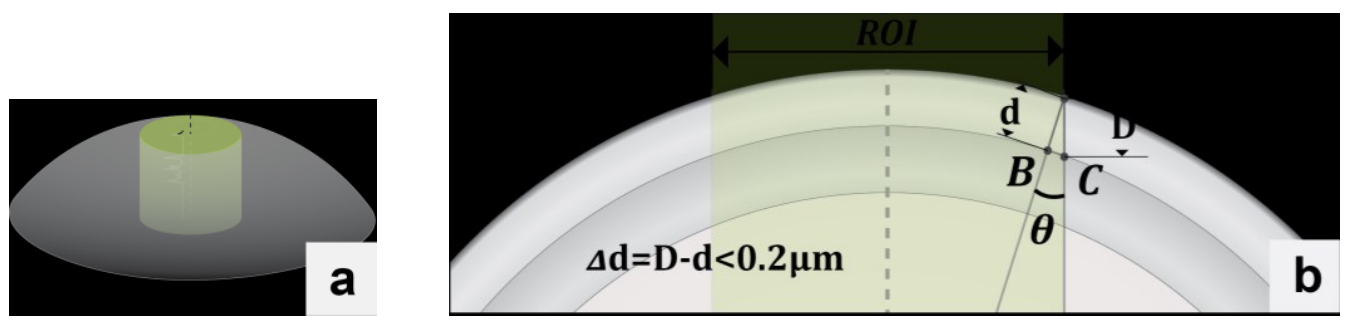
Figure 5 (a) the surface model of a two-layer pellet used. The cylinder at the centre illustrates the ROI where 200 neighboring tomography signals were selected. (b) a cross-section model of the pellet with a diameter of $850 \mu \mathrm{m}$. The OCT measured area covers $700 \mu \mathrm{m} \times 700 \mu \mathrm{m}$ and the ROI has a diameter of $68 \mu \mathrm{m}$. D is the OCT-measured thickness of the first coating layer at the edge of ROI. $\mathbf{d}$ is the true thickness of the first coating layer. If $\mathbf{d}$ has a thickness of $40 \mu m$, the thickness error (e.g., the difference between $\mathrm{d}$ and $\mathrm{D}$ ) is smaller than $0.2 \mu \mathrm{m}$.

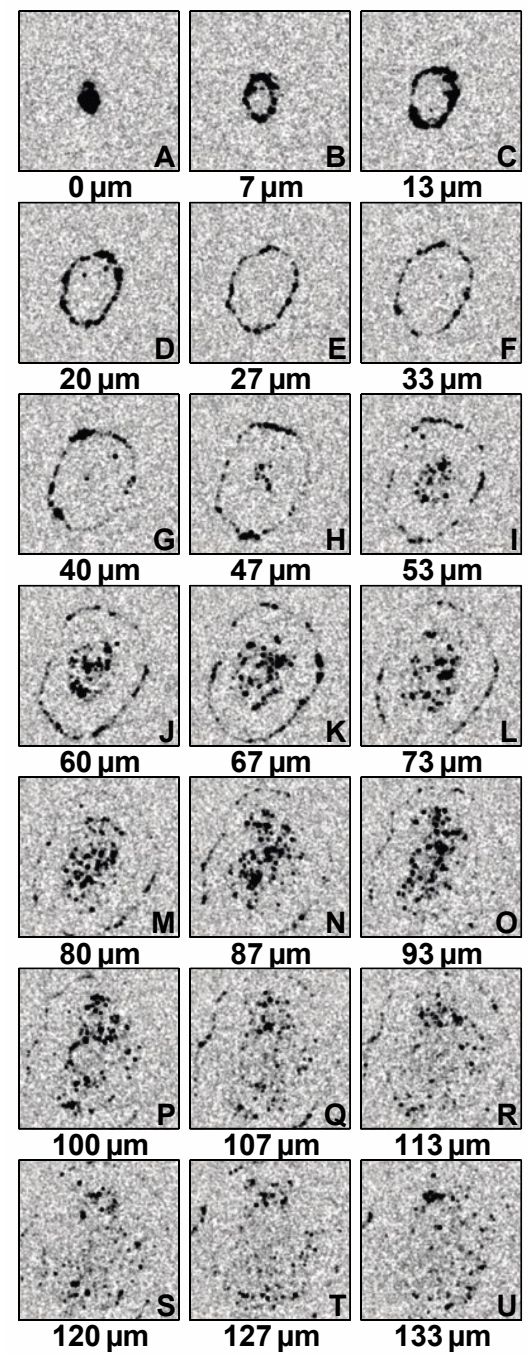

(a)
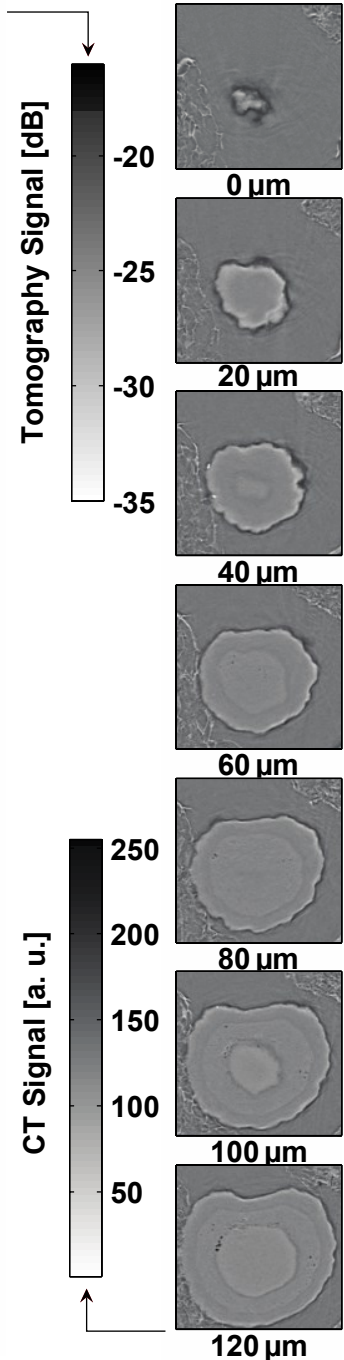

(b)

Figure 6: (a) En-face images represented in decibel with $6.7 \mu m$ depth interval between successive images. Each image covers a field of $700 \mu m \times 700 \mu m$ (b) $\mathrm{X} \mu \mathrm{CT}$ depth-resolved en-face images for the same sample (through a slightly different plane of the pellet). 
As a further illustration of the microstructure of the pellet Figure 6 (a) shows a set of en-face images at progressive depths reconstructed for the pellet sample. Again the dark areas correspond to the region where there is a change in the refractive index, e.g., at the interface between two coatings or between two different materials (particle). Because of the surface curvature of the spherical pellet sample, one can see clearly the annular shape (in dark) in image A to F. In image $G$ a dark dot reappeared at the centre, indicating the inner coating layer starts to appear. The thickness of outer coating layer could then be estimated as $40 \mu \mathrm{m}$ (image A to G). In a similar manner, the thickness of the inner coating layer could also be estimated as $47 \mu m$ (image $\mathrm{G}$ to $\mathrm{N}$ ). As a comparison, Figure 6 (b) displays seven $\mathrm{X} \mu \mathrm{CT}$ en-face images of the same pellet sample at corresponding depths. Again there is a general agreement between the FF-OCT and the $\mathrm{X} \mu \mathrm{CT}$ results.

Both Figures 3 (a) (b) and 6 (a) revealed that there are particle-like features within the inner coating layer and that these features have a wide range of particle size. In order to characterise the achieved lateral resolution of the developed FF-OCT system, a calibration specimen (stage micrometre, R1L3S2P, Thorlabs) was measured under the same experimental settings. Figure 7 shows the extracted surface height profile. The FWHM of the scale bar was measured to be $12 \mu \mathrm{m}$. The true width of the scale bar is about $5 \mu \mathrm{m}$ as revealed by a more precise measurement using a high-resolution optical microscope. The lateral resolution of the presented OCT system, defined as the point spread function, is finally calculated as $11 \mu \mathrm{m}$ through a de-convolution process [16].

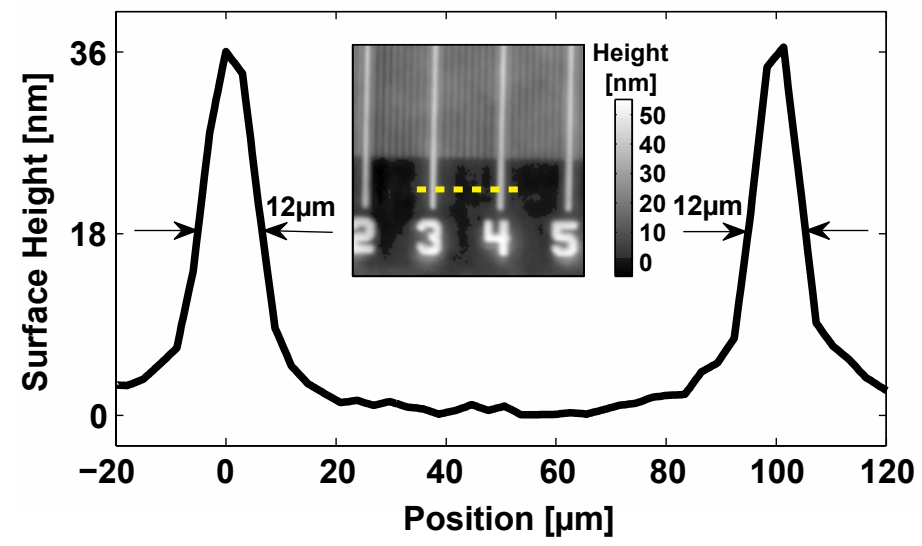

Figure 7: Surface height profile of two scale marks with a resolved FWHM of $12 \mu \mathrm{m}$. The inset shows the surface height image of the stage micrometre. 
We note that Pygall [17] and Laksmana [18] evaluated pellet coatings using confocal laser scanning microscopy (CLSM), which is a single point imaging technique with depth selectivity. Both CLSM and OCT can provide high-resolution cross-sectional images of a sample. As a conventional microscopy technique, CLSM detects the variation of optical intensity of the reflected/scattered light, while OCT detects the interference patterns (e.g., the electric field of the light reflected/scattered from the reference and sample arms) [19] thus has a better imaging depth and is more sensitive to small changes in the inner structures/properties of a sample.

Terahertz imaging has also been evaluated for directly measuring the layer thickness of film coatings [8-10]. Since most pharmaceutical excipients used in film coating are either transparent or semi-transparent in the terahertz frequency range [4], TPI can be used to quantify thick pharmaceutical coatings in the range of $40-140 \mu m$ and beyond [11]. However, due to the relatively long wavelength of terahertz radiation $(300 \mu m$ at $1 \mathrm{THz})$, TPI has limited spatial resolution in lateral direction and thus is not suitable to investigate the fine structure of small sized pellet samples such as the ones studied here. In contrast, the FF-OCT system presented in this study achieved a spatial resolution of $11 \mu \mathrm{m} \times 3.6 \mu \mathrm{m}$ (lateral $\times$ axial ) allowing a more precise analysis of the coating thickness and revealing more detailed information on the inner structures of a sample. This makes the FF-OCT an attractive tool for non-destructive evaluation of small pharmaceutical pellets. We also demonstrated in this work that $\mathrm{X} \mu \mathrm{CT}$ has excellent capability in obtaining the high-resolution 3D inner structure of a pellet sample non-destructively. However, currently the $\mathrm{X} \mu \mathrm{CT}$ measurement and the required subsequent image reconstruction is a time-consuming process (both on the order of hours for the $\mathrm{X} \mu \mathrm{CT}$ results presented here). Furthermore, the ionising nature of X-ray radiation also makes it extremely difficult, if not impossible, to be used for online monitoring applications. In this sense, the FF-OCT method proposed here is advantageous as it is fast, compact and safe (it uses low power near infrared light) thus it could be used for both offline and online applications.

\section{CONCLUSIONS}

In this study, we have demonstrated the capability of FF-OCT for non-destructive evaluation of film coatings applied to spherical pellet of less than $1 \mathrm{~mm}$ diameter. By utilising the axial resolution of $3.6 \mu \mathrm{m}$, the layer thickness could be precisely determined as $40 \mu \mathrm{m}$ and 
$50 \mu \mathrm{m}$ for the outer and inner coatings of the pellet sample, respectively. The achieved lateral resolution is $11 \mu \mathrm{m}$ and this allows particle like features within the inner coating layer to be resolved. This, together with its low cost and ease of use, makes the presented FF-OCT an attractive analytical tool for the non-destructive evaluation of the coating thickness and internal structure of pharmaceutical pellets.

\section{ACKNOWLEDGEMENT}

This work is partially supported by the Engineering and Physical Sciences Research Council (EPSRC, EP/K031511/1 and EP/K031511/1). The authors would like to acknowledge Neil Turnbull from Pfizer UK for supplying samples of coated pellets used in this work.

\section{References}

1. Basit AW, Podczeck F, Newton JM, Waddington WA, Ell PJ, Lacey LF 2004. The use of formulation technology to assess regional gastrointestinal drug absorption in humans. Eur J Pharm Sci 21:179-189.

2. Ho L, Cuppok Y, Muschert S, Gordon KC, Pepper M, Shen Y, Siepmann F, Siepmann J, Taday PF, Rades T 2009. Effects of film coating thickness and drug layer uniformity on in vitro drug release from sustained-release coated pellets: A case study using terahertz pulsed imaging. Int J Pharm 382:151-159.

3. Ringqvist A, Taylor LS, Ekelund K, Ragnarsson G, Engström S, Axelsson A 2003. Atomic force microscopy analysis and confocal Raman microimaging of coated pellets. Int J Pharm 267:35-47.

4. Ho L, Müller R, Gordon KC, Kleinebudde P, Pepper M, Rades T, Shen Y, Taday PF, Zeitler JA 2008. Applications of terahertz pulsed imaging to sustained-release tablet film coating quality assessment and dissolution performance. J Control Release 127:79-87.

5. Andersson M, Holmquist B, Lindquist J, Nilsson O, Wahlund K-G 2000. Analysis of film coating thickness and surface area of pharmaceutical pellets using fluorescence microscopy and image analysis. J Pharm Biomed Anal 22:325-339.

6. Brock D, Zeitler JA, Funke A, Knop K, Kleinebudde, P 2012. A comparison of quality control methods for active coating processes. Int J Pharm 439:289-295.

7. Reich G 2005. Near-infrared spectroscopy and imaging: Basic principles and pharmaceutical applications. Adv Drug Deliv Rev 57:1109-1143. 
8. Shen Y, Taday PF 2008. Development and Application of Terahertz Pulsed Imaging for Non-destructive Inspection of Pharmaceutical Tablet. IEEE J Sel Top Quant Electron 14:407-415.

9. Zeitler JA, Taday PF, Newnham DA, Pepper M, Gordon KC, Rades T 2007. Terahertz pulsed spectroscopy and imaging in the pharmaceutical setting - a review. J Pharm Pharmacol 59:209-223.

10.Haaser M, Karrout Y, Velghe C, Cuppok Y, Gordon K, Pepper M, Siepmann J, Strachan C, Taday PF, Rades T. Proceedings of the 2nd Electronic Conference on Pharmaceutical Sciences, 2012.

11.Zhong S, Shen Y, Ho L, May RK, Zeitler JA, Evans M, Taday PF, Pepper M, Rades T, Gordon KC, Müller R, Kleinebudde P 2011. Non-destructive quantification of pharmaceutical tablet coatings using terahertz pulsed imaging and optical coherence tomography. Opt Laser Eng 49:361-365.

12.Koller DM, Hannesschläger G, Leitner M, Khinast JG 2011. Non-destructive analysis of tablet coatings with optical coherence tomography. Eur J Pharm Sci 44:142-148.

13.Mauritz JM, Morrisby RS, Hutton RS, Legge CH, Kaminski CF 2010. Imaging pharmaceutical tablets with optical coherence tomography. J Pharm Sci 99:385-391.

14.Larkin KG 1996. Efficient nonlinear algorithm for envelope detection in white light interferometry. J Opt Soc Am A 13:832-843.

15.Dubois A, Boccara AC. 2008. Full-field optical coherence tomography. In Drexler W, Fujimoto JG, editors. Optical Coherence Tomography, ed.: Springer Berlin Heidelberg. p 565-591.

16.Andrews HC, Tescher AG, Kruger RP 1972. Image processing by digital computer. Spectrum IEEE 9:20-32.

17.Pygall SR, Whetstone J, Timmins P, Melia CD 2007. Pharmaceutical applications of confocal laser scanning microscopy: The physical characterisation of pharmaceutical systems. Adv Drug Deliv Rev 59:1434-1452.

18.Laksmana FL, Van Vliet LJ, Hartman Kok PJ, Vromans H, Frijlink HW, Van der Voort Maarschalk K 2009. Quantitative Image Analysis for Evaluating the Coating Thickness and Pore Distribution in Coated Small Particles. Pharm Res 26:965-976. 
19.Izatt JA, Choma MA. 2008. Theory of optical coherence tomography. In Drexler W, Fujimoto JG, editors. Optical Coherence Tomography, ed.: Springer Berlin Heidelberg. p 47-72. 